\title{
Association of genetic variants with ischemic stroke in Japanese individuals with or without metabolic syndrome
}

\author{
TETSURO YOSHIDA ${ }^{1}$, KIMIHIKO KATO ${ }^{2}$, KIYOSHI YOKOI $^{2}$, MITSUTOSHI OGURI $^{3}$, \\ SACHIRO WATANABE ${ }^{4}$, NORIFUMI METOKI ${ }^{5}$, HIDEMI YOSHIDA ${ }^{6}$, KEI SATOH ${ }^{6}$, \\ YUKITOSHI AOYAGI ${ }^{7}$, YOSHINORI NOZAWA ${ }^{8}$ and YOSHIJI YAMADA ${ }^{9}$
}

\author{
${ }^{1}$ Department of Cardiovascular Medicine, Inabe General Hospital, Inabe; ${ }^{2}$ Department of Cardiovascular Medicine, \\ Gifu Prefectural Tajimi Hospital, Tajimi; ${ }^{3}$ Department of Cardiology, Japanese Red Cross Nagoya First Hospital, \\ Nagoya; ${ }^{4}$ Department of Cardiology, Gifu Prefectural General Medical Center, Gifu; ${ }^{5}$ Department of Internal Medicine, \\ Hirosaki Stroke Center, Hirosaki; ${ }^{6}$ Department of Vascular Biology, Institute of Brain Science, Hirosaki University \\ Graduate School of Medicine, Hirosaki; ${ }^{7}$ Department of Genomics for Longevity and Health, \\ Tokyo Metropolitan Institute of Gerontology, Tokyo; ${ }^{8}$ Gifu International Institute of Biotechnology \\ and Tokai Gakuin University, Kakamigahara; ${ }^{9}$ Department of Human Functional Genomics, \\ Life Science Research Center, Mie University, Tsu, Japan
}

Received September 7, 2009; Accepted October 21, 2009

DOI: 10.3892/ijmm_00000342

\begin{abstract}
Although metabolic syndrome has been recognized as a risk factor for ischemic stroke, genetic factors associated with ischemic stroke in individuals with metabolic syndrome remain unknown. We examined an association of genetic variants with ischemic stroke among individuals with or without metabolic syndrome. The study population comprised 4,387 unrelated Japanese individuals, including 1,884 individuals with metabolic syndrome ( 240 subjects with ischemic stroke and 1,644 controls) and 2,503 individuals without metabolic syndrome (280 subjects with ischemic stroke and 2,223 controls). The 150 polymorphisms examined in the present study were selected by genome-wide association studies of ischemic stroke and myocardial infarction with the use of the GeneChip Human Mapping 500K Array Set (Affymetrix). The initial chi-square test revealed that the $\mathrm{C} \rightarrow \mathrm{T}$ polymorphism (rs9925481) of CLEC16A and the $\mathrm{A} \rightarrow \mathrm{G}$ polymorphism (rs4923918) of SPTBN5 were significantly $(\mathrm{P}<0.005)$ associated with ischemic stroke among individuals with metabolic syndrome. No polymorphism was significantly associated with ischemic stroke among individuals without metabolic syndrome. Multivariable logistic regression analysis with adjustment for covariates and a stepwise forward selection procedure revealed that the $\mathrm{A} \rightarrow \mathrm{G}$ polymorphism (rs4923918)
\end{abstract}

Correspondence to: Dr Yoshiji Yamada, Department of Human Functional Genomics, Life Science Research Center, Mie University, 1577 Kurima-machiya, Tsu, Mie 514-8507, Japan E-mail: yamada@gene.mie-u.ac.jp

Key words: genetics, polymorphism, ischemic stroke, cerebral infarction, metabolic syndrome of SPTBN5 was significantly $(\mathrm{P}<0.005)$, and the $\mathrm{C} \rightarrow \mathrm{T}$ polymorphism (rs9925481) of CLEC16A was almost significantly, associated with ischemic stroke in individuals with metabolic syndrome. Genetic variants that confer susceptibility to ischemic stroke may differ among individuals with or without metabolic syndrome. Stratification of subjects according to the presence or absence of metabolic syndrome may thus be important for personalized prevention of ischemic stroke based on genetic information.

\section{Introduction}

Metabolic syndrome is defined by a clustering of abdominal obesity, an increased serum concentration of triglycerides, a decreased serum concentration of high density lipoprotein (HDL)-cholesterol, high blood pressure, and an increased fasting blood glucose level, in which insulin resistance may be the major underlying cause of this condition (1-3). In addition, various environmental and genetic factors (4-8) contribute to individual susceptibility to each component of metabolic syndrome. Although metabolic syndrome has been recognized as a risk factor for coronary heart disease $(9,10)$ and ischemic stroke (11-15), genetic variants that confer susceptibility to ischemic stroke in individuals with or without metabolic syndrome have remained unknown. Given that stroke is the leading cause of severe disability and the third leading cause of death, after heart disease and cancer, in Western countries and Japan (16), the identification of biomarkers for stroke risk is important both for risk prediction and for intervention to avert future events.

We previously showed that several gene polymorphisms were associated with atherothrombotic cerebral infarction in individuals with metabolic syndrome (17). To further examine whether the association of polymorphisms with ischemic stroke is influenced by the presence or absence of metabolic 
syndrome, we have performed an association study for 150 polymorphisms of 144 candidate genes and ischemic stroke in 4,387 Japanese individuals with or without metabolic syndrome. The purpose of the present study was to identify genetic variants that confer susceptibility to ischemic stroke in Japanese individuals with or without metabolic syndrome independently and thereby to assess the genetic risk of ischemic stroke in such individuals separately.

\section{Materials and methods}

Study population. The study population comprised 4,387 unrelated Japanese individuals who either visited outpatient clinics of or were admitted to one of the participating hospitals (Gifu Prefectural General Medical Center and Gifu Prefectural Tajimi Hospital in Gifu Prefecture, Japan; and Hirosaki University Hospital, Reimeikyo Rehabilitation Hospital, and Hirosaki Stroke Center in Aomori Prefecture, Japan) between October 2002 and March 2008 because of various symptoms or for an annual health checkup, or who were recruited to a population-based prospective cohort study of aging and agerelated diseases in Nakanojo, Gunma Prefecture, Japan.

Diagnosis of metabolic syndrome was based on a modified version of the definition of metabolic syndrome proposed by the American Heart Association and US National Heart, Lung, and Blood Institute (3). In this modified version, which was also used in the West of Scotland Coronary Prevention Study (18) and the Women's Health Study (19), body mass index (BMI) replaces waist circumference. On the basis of the recent recognition of a need to revise BMI criteria for obesity in Japanese and other Asian populations (20), we set the cutoff point for obesity as a BMI of $\geq 25 \mathrm{~kg} / \mathrm{m}^{2}$. A total of 1,884 subjects with metabolic syndrome had thus three or more of the following five components: (i) a BMI of $\geq 25 \mathrm{~kg} / \mathrm{m}^{2}$; (ii) a serum concentration of triglycerides of $\geq 1.65 \mathrm{mmol} / \mathrm{l}$ $(150 \mathrm{mg} / \mathrm{dl})$ or drug treatment for elevated triglycerides; (iii) a serum concentration of HDL-cholesterol of $<1.04 \mathrm{mmol} / 1$ $(40 \mathrm{mg} / \mathrm{dl})$ for men or $<1.30 \mathrm{mmol} / 1(50 \mathrm{mg} / \mathrm{dl})$ for women, or drug treatment for reduced HDL-cholesterol; (iv) a systolic blood pressure of $\geq 130 \mathrm{mmHg}$ or diastolic blood pressure of $\geq 85 \mathrm{mmHg}$, or drug treatment for hypertension; and (v) a fasting plasma glucose level of $\geq 5.50 \mathrm{mmol} / \mathrm{l}(100 \mathrm{mg} / \mathrm{dl})$ or drug treatment for elevated glucose. A total of 3,867 subjects without metabolic syndrome had none or one of the above five components, subjects who had two components were excluded from the present study.

Among 1,884 subjects with metabolic syndrome, 240 individuals (154 men, 86 women) had ischemic stroke, and among 2,503 subjects without metabolic syndrome, 280 individuals (145 men, 135 women) had ischemic stroke. The diagnosis of ischemic stroke was based on the occurrence of a new and abrupt focal neurological deficit, with neurological symptoms and signs persisting for $>24 \mathrm{~h}$; it was confirmed by positive findings in computed tomography or magnetic resonance imaging (or both) of the head. The type of stroke was determined according to the Classification of Cerebrovascular Diseases III (21). Individuals with cardiogenic embolic infarction, lacunar infarction alone, transient ischemic attack, moyamoya disease, or cerebral venous sinus thrombosis were excluded from the study, as were those with atrial fibrillation

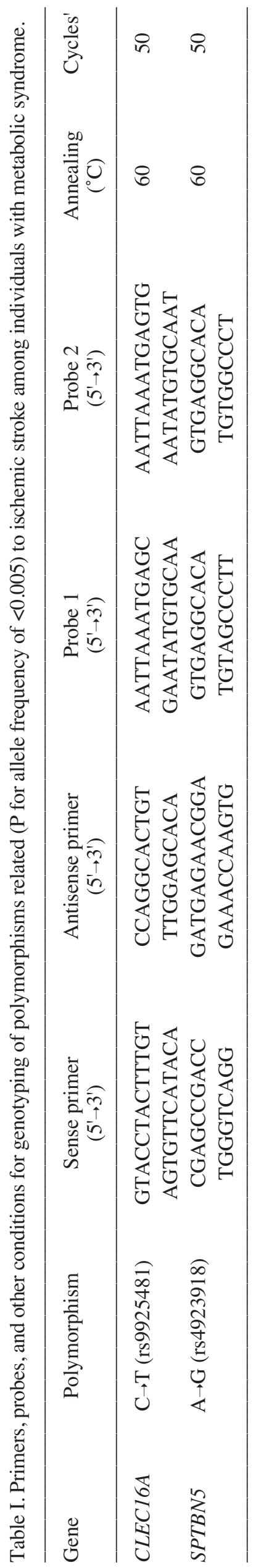


Table II. Characteristics of subjects with ischemic stroke and controls among individuals with or without metabolic syndrome.

\begin{tabular}{|c|c|c|c|c|c|c|}
\hline \multirow[b]{2}{*}{ Characteristic } & \multicolumn{3}{|c|}{ With metabolic syndrome } & \multicolumn{3}{|c|}{ Without metabolic syndrome } \\
\hline & Ischemic stroke & Controls & $\mathrm{P}$ & Ischemic stroke & Controls & $\mathrm{P}$ \\
\hline No. of subjects & 240 & 1644 & & 280 & 2223 & \\
\hline Age (years) & $68.1 \pm 8.6$ & $64.7 \pm 10.1$ & $<0.0001$ & $68.9 \pm 11.8$ & $63.7 \pm 12.3$ & $<0.0001$ \\
\hline Sex (male/female, \%) & $64.2 / 35.8$ & $65.2 / 34.8$ & 0.7526 & $51.8 / 48.2$ & $47.1 / 52.9$ & 0.1354 \\
\hline $\begin{array}{l}\text { Body mass index } \\
\left(\mathrm{kg} / \mathrm{m}^{2}\right)\end{array}$ & $24.5 \pm 3.7$ & $25.2 \pm 3.5$ & 0.0002 & $21.6 \pm 2.2$ & $21.9 \pm 2.7$ & 0.7051 \\
\hline $\begin{array}{l}\text { Current or former } \\
\text { smoker }(\%)\end{array}$ & 21.3 & 24.4 & 0.2813 & 4.6 & 13.6 & $<0.0001$ \\
\hline Hypertension (\%) & 91.3 & 82.4 & 0.0002 & 41.2 & 25.2 & $<0.0001$ \\
\hline $\begin{array}{l}\text { Systolic blood } \\
\text { pressure (mmHg) }\end{array}$ & $159 \pm 29$ & $148 \pm 26$ & $<0.0001$ & $138 \pm 26$ & $130 \pm 23$ & 0.0104 \\
\hline $\begin{array}{l}\text { Diastolic blood } \\
\text { pressure }(\mathrm{mmHg})\end{array}$ & $87 \pm 18$ & $79 \pm 15$ & $<0.0001$ & $78 \pm 16$ & $74 \pm 13$ & 0.0494 \\
\hline Hypercholesterolemia (\%) & 38.8 & 34.7 & 0.2193 & 11.4 & 18.0 & 0.0044 \\
\hline $\begin{array}{l}\text { Serum total cholesterol } \\
(\mathrm{mmol} / \mathrm{l})\end{array}$ & $5.35 \pm 1.08$ & $5.32 \pm 1.13$ & 0.5314 & $4.95 \pm 0.71$ & $5.08 \pm 0.90$ & 0.2642 \\
\hline $\begin{array}{l}\text { Serum triglyceride } \\
(\mathrm{mmol} / \mathrm{l})\end{array}$ & $2.06 \pm 1.18$ & $2.18 \pm 1.46$ & 0.3687 & $1.03 \pm 0.31$ & $1.08 \pm 0.48$ & 0.8490 \\
\hline $\begin{array}{l}\text { Serum HDL-cholesterol } \\
(\mathrm{mmol} / \mathrm{l})\end{array}$ & $1.19 \pm 0.37$ & $1.14 \pm 0.32$ & 0.0561 & $1.45 \pm 0.33$ & $1.60 \pm 0.38$ & 0.0040 \\
\hline Diabetes mellitus (\%) & 64.2 & 53.2 & 0.0013 & 7.5 & 5.0 & 0.0995 \\
\hline $\begin{array}{l}\text { Fasting plasma glucose }) \\
(\mathrm{mmol} / \mathrm{l}\end{array}$ & $7.65 \pm 2.83$ & $7.89 \pm 3.73$ & 0.5532 & $5.21 \pm 1.46$ & $5.34 \pm 1.81$ & 0.3749 \\
\hline $\begin{array}{l}\text { Blood glycosylated } \\
\text { hemoglobin }(\%)\end{array}$ & $6.50 \pm 1.63$ & $6.73 \pm 1.93$ & 0.3746 & $5.10 \pm 0.63$ & $5.32 \pm 0.95$ & 0.0965 \\
\hline
\end{tabular}

Quantitative data are means \pm SD. HDL, high density lipoprotein.

in the absence or presence of valvular heart disease. The 3,867 control subjects had no history of ischemic or hemorrhagic stroke or other cerebral diseases; of coronary heart disease or peripheral arterial occlusive disease; or of other atherosclerotic, thrombotic, embolic, or hemorrhagic disorders.

The study protocol complied with the Declaration of Helsinki and was approved by the Committees on the Ethics of Human Research of Mie University Graduate School of Medicine, Hirosaki University Graduate School of Medicine, Gifu International Institute of Biotechnology, Tokyo Metropolitan Institute of Gerontology, and participating hospitals. Written informed consent was obtained from each participant.

Selection of polymorphisms. Our aim was to identify genetic variants associated with ischemic stroke in Japanese individuals with or without metabolic syndrome in a case-control association study. The 150 polymorphisms examined in the present study (data not shown) were selected by genome-wide association studies of ischemic stroke and myocardial infarction (P-value for allele frequency $<1.0 \times 10^{-7}$ ) with the use of the GeneChip Human Mapping 500K Array Set (Affymetrix, Santa Clara, CA) (22). We did not examine the relation of these polymorphisms to ischemic stroke among individuals with metabolic syndrome in our previous study (17).
Genotyping of polymorphisms. Venous blood (7 ml) was collected in tubes containing $50 \mathrm{mmol} / \mathrm{l}$ ethylenediaminetetraacetic acid (disodium salt), and genomic DNA was isolated with a kit (Genomix; Talent, Trieste, Italy). Genotypes of the 150 polymorphisms were determined at G\&G Science (Fukushima, Japan) by a method that combines the polymerase chain reaction and sequence-specific oligonucleotide probes with suspension array technology (Luminex, Austin, TX). Primers, probes, and other conditions for genotyping of polymorphisms related to ischemic stroke are shown in Table I. Detailed genotyping methodology was described previously (23).

Statistical analysis. Quantitative data were compared between subjects with ischemic stroke and controls by the unpaired Student's t-test. Categorical data were compared by the chi-square test. Allele frequencies were estimated by the gene counting method, and the chi-square test was used to identify departures from Hardy-Weinberg equilibrium. In the initial screen, the allele frequencies of each polymorphism were compared between subjects with ischemic stroke and controls by the chi-square test. Polymorphisms with a P-value for allele frequency of $<0.005$ were further examined by multivariable logistic regression analysis with adjustment for covariates. Multivariable logistic regression analysis was thus 
Table III. Genotype distributions of polymorphisms significantly (P-value for allele frequency <0.005) associated with ischemic stroke among individuals with or without metabolic syndrome as determined by the chi-square test.

\begin{tabular}{|c|c|c|c|c|c|}
\hline Gene & Polymorphism & $\mathrm{dbSNP}$ & Ischemic stroke ${ }^{a}$ & Controls ${ }^{\mathrm{a}}$ & $\mathrm{P}$ (allele frequency) \\
\hline \multicolumn{6}{|c|}{ With metabolic syndrome } \\
\hline \multirow[t]{4}{*}{ CLEC16A } & $\mathrm{C} \rightarrow \mathrm{T}$ & rs9925481 & & & 0.0005 \\
\hline & $\mathrm{CC}$ & & $166(69.5)$ & $1289(78.8)$ & \\
\hline & $\mathrm{CT}$ & & $66(27.6)$ & $327(20.0)$ & \\
\hline & TT & & $7 \quad(2.9)$ & $20(1.2)$ & \\
\hline \multirow[t]{4}{*}{ SPTBN5 } & $A \rightarrow G$ & rs4923918 & & & 0.0013 \\
\hline & AA & & $12(5.0)$ & $28 \quad(1.7)$ & \\
\hline & $\mathrm{AG}$ & & $70(29.3)$ & 405 (24.7) & \\
\hline & GG & & $157(65.7)$ & $1205(73.6)$ & \\
\hline
\end{tabular}

${ }^{a}$ Numbers in parentheses are percentages.

performed with ischemic stroke as a dependent variable and independent variables including age, sex (0, woman; 1 , man), BMI, smoking status ( 0 , nonsmoker; 1 , smoker), history of hypertension, diabetes mellitus, and hypercholesterolemia $(0$, no history; 1 , positive history), and genotype of each polymorphism; and the P-value, odds ratio, and 95\% confidence interval were calculated. Each genotype was assessed according to dominant, recessive, and additive genetic models. Additive models included the additive 1 (heterozygotes versus wild-type homozygotes) and additive 2 (variant homozygotes versus wild-type homozygotes) models, which were analyzed simultaneously with a single statistical model. We also performed a stepwise forward selection procedure to examine the effects of genotypes as well as of other covariates on ischemic stroke; each genotype was examined according to a dominant or recessive model on the basis of statistical significance in the multivariable logistic regression analysis. The P-levels for inclusion in and exclusion from the model were 0.25 and 0.1 , respectively. Given the multiple comparisons of genotypes with ischemic stroke, we adopted the criterion of a P-value of $<0.005$ for statistical significance of association. For other clinical background data, a P-value of $<0.05$ was considered statistically significant. Statistical significance was examined by two-sided tests performed with JMP version 6.0 and JMP Genomics version 3.2 software (SAS Institute, Cary, NC).

\section{Results}

The characteristics of the 4,387 study subjects are shown in Table II. For individuals with metabolic syndrome, age, systolic and diastolic blood pressure, and the prevalence of hypertension and diabetes mellitus were greater, whereas BMI was lower, in subjects with ischemic stroke than in controls. For individuals without metabolic syndrome, age, systolic and diastolic blood pressure, and the prevalence of hypertension were greater, whereas the percentage of smokers, the prevalence of hypercholesterolemia, and the serum concentration of HDL-cholesterol were lower, in subjects with ischemic stroke than in controls.
Comparison of allele frequencies by the chi-square test revealed that the $\mathrm{C} \rightarrow \mathrm{T}$ polymorphism (rs9925481) of CLEC16A and the $\mathrm{A} \rightarrow \mathrm{G}$ polymorphism (rs4923918) of SPTBN5 were significantly $(\mathrm{P}<0.005)$ associated with ischemic stroke in individuals with metabolic syndrome (Table III). No polymorphism was significantly related to ischemic stroke in individuals without metabolic syndrome.

Multivariable logistic regression analysis with adjustment for age, sex, BMI, smoking status, and the prevalence of hypertension, hypercholesterolemia, and diabetes mellitus revealed that the $\mathrm{C} \rightarrow \mathrm{T}$ polymorphism (rs9925481) of CLEC16A (dominant model) and the $\mathrm{A} \rightarrow \mathrm{G}$ polymorphism (rs4923918) of SPTBN5 (dominant, recessive, and additive 2 models) were significantly $(\mathrm{P}<0.005)$ associated with ischemic stroke in individuals with metabolic syndrome (Table IV).

A stepwise forward selection procedure was performed to examine the effects of genotypes for the polymorphisms related to ischemic stroke by the chi-square test as well as of age, sex, BMI, smoking status, and the prevalence of hypertension, hypercholesterolemia, and diabetes mellitus on ischemic stroke (Table V). Age, hypertension, and SPTBN5 genotype (dominant model), in descending order of statistical significance, were significant $(\mathrm{P}<0.005)$ and independent determinants of ischemic stroke. CLEC 16A genotype (dominant model) was an almost significant $(\mathrm{P}=0.0053)$ and independent determinant of ischemic stroke.

Finally, we examined whether the genotype distributions for the polymorphisms associated with ischemic stroke among individuals with metabolic syndrome were in Hardy-Weinberg equilibrium. The genotype distributions for the $A \rightarrow G$ polymorphism of SPTBN5 (subjects with ischemic stroke, $\mathrm{P}=0.2589$; controls, $\mathrm{P}=0.3647$ ) and the $\mathrm{C} \rightarrow \mathrm{T}$ polymorphism of CLEC16A (subjects with ischemic stroke, $\mathrm{P}=0.8873$; controls, $\mathrm{P}=0.8851$ ) were in Hardy-Weinberg equilibrium both in subjects with ischemic stroke and in controls.

\section{Discussion}

We examined the possible relations of 150 polymorphisms of 144 candidate genes to the prevalence of ischemic stroke in 
Table V. Effects of genotypes and other characteristics on ischemic stroke among individuals with or without metabolic syndrome determined by a stepwise forward selection procedure $(\mathrm{P}<0.005)$.

\begin{tabular}{lrc}
\hline Variable & $\mathrm{P}$ & $\mathrm{R}^{2}$ \\
\hline With metabolic syndrome & & \\
Age & $<0.0001$ & 0.0180 \\
Hypertension & 0.0008 & 0.0081 \\
SPTBN5 (AG+GG vs AA) & 0.0046 & 0.0072 \\
CLEC16A (CT+TT vs CC) & 0.0053 & 0.0055 \\
\hline
\end{tabular}

$\mathrm{R}^{2}$, contribution rate.

individuals with or without metabolic syndrome. Our association study with three steps of analysis (chi-square test, multivariable logistic regression analysis with adjustment for covariates, and stepwise forward selection procedure) revealed that the A $\rightarrow$ G polymorphism (rs4923918) of SPTBN5 was significantly, and the $\mathrm{C} \rightarrow \mathrm{T}$ polymorphism (rs9925481) of CLEC16A was almost significantly, associated with ischemic stroke in Japanese individuals with metabolic syndrome.

Spectrin consists of $\alpha$ and $\beta$ subunits and is the predominant component of the membrane skeleton of the red cell. Spectrin is essential in determining the properties of the membrane including its shape and deformability (24). Based on structural features, spectrin, $B$, non-erythrocytic 5 (SPTBN5) is likely to form heterodimers and oligomers with $\alpha$-spectrin and to interact directly with cellular membranes (25). Polymorphisms of SPTBN5 have not been associated with human disease. We have now shown that the $\mathrm{A} \rightarrow \mathrm{G}$ polymorphism (rs4923918) in intron 32 of SPTBN5 was significantly associated with the prevalence of ischemic stroke in individuals with metabolic syndrome, with the $\mathrm{G}$ allele protecting against this condition. The effect of this polymorphism on membrane properties might account for its relation to ischemic stroke, although the underlying mechanism remains unclear.

The C-type lectin domain family 16, member A (CLEC16A) was shown to be expressed in B lymphocytes and natural killer and dendritic cells (26). The $\mathrm{G}$ allele of the $\mathrm{G} \rightarrow \mathrm{A}$ polymorphism of CLEC16A (rs2903692) was related to an increased risk for type 1 diabetes mellitus in Japanese subjects (27), as well as in white populations $(28,29)$. Three common non-coding variants of CLEC16A (rs2903692, rs725613, and rs17673553), which were in linkage disequilibrium, were significantly associated with type 1 diabetes mellitus in a genome-wide association study (29). These results suggest that CLEC16A is involved in the pathogenesis of type 1 diabetes mellitus. We have now shown that $\mathrm{C} \rightarrow \mathrm{T}$ polymorphism (rs9925481) in intron 11 of CLEC16A was almost significantly associated with ischemic stroke in individuals with metabolic syndrome, with the $\mathrm{T}$ allele representing a risk factor for this condition. The relation of this polymorphism to type 1 diabetes mellitus in our population remains unclear.

Our study has several limitations: (i) It is possible that one or more of the polymorphisms associated with ischemic stroke in the present study are in linkage disequilibrium with 
other polymorphisms in the same gene or in other nearby genes that are actually responsible for the development of this condition; (ii) The functional relevance of the identified polymorphisms to gene transcription or to protein structure or function was not determined in the present study; (iii) Although we adopted the criterion of $\mathrm{P}<0.005$ for association to compensate for the multiple comparisons of genotypes with ischemic stroke, it is not possible to exclude completely potential statistical errors such as false positives; (iv) The results of the present study were not replicated in independent subject panels.

In conclusion, our present results suggest that SPTBN5 and CLEC16A are susceptibility loci for ischemic stroke among Japanese individuals with metabolic syndrome. Genotypes for these polymorphisms may prove informative for assessment of genetic risk for ischemic stroke in such individuals. Genetic variants that confer susceptibility to ischemic stroke may differ among individuals with or without metabolic syndrome. Stratification of subjects according to the presence or absence of metabolic syndrome may thus be important for personalized prevention of ischemic stroke based on genetic information. Given that our present study may be considered as hypothesis generating, validation of our findings will require their replication with independent subject panels.

\section{Acknowledgements}

This work was supported in part by Grants-in-Aid for Scientific Research from the Ministry of Education, Culture, Sports, Science, and Technology of Japan (nos. 18209023, 18018021, and 19659149 to Y.Y.).

\section{References}

1. Expert Panel on Detection, Evaluation, and Treatment of High Blood Cholesterol in Adults: Executive Summary of The Third Report of The National Cholesterol Education Program (NCEP) Expert Panel on Detection, Evaluation, And Treatment of High Blood Cholesterol In Adults (Adult Treatment Panel III). JAMA 285: 2486-2497, 2001.

2. National Cholesterol Education Program (NCEP) Expert Panel on Detection, Evaluation, and Treatment of High Blood Cholesterol in Adults (Adult Treatment Panel III): Third Report of the National Cholesterol Education Program (NCEP) Expert Panel on Detection, Evaluation, and Treatment of High Blood Cholesterol in Adults (Adult Treatment Panel III) final report. Circulation 106: 3143-3421, 2002.

3. Grundy SM, Cleeman JI, Daniels SR, et al: Diagnosis and management of the metabolic syndrome: an American Heart Association/National Heart, Lung, and Blood Institute Scientific Statement. Circulation 112: 2735-2752, 2005.

4. Yamada Y, Kato K, Kameyama T, et al: Genetic factors for obesity. Int J Mol Med 18: 843-851, 2006.

5. Yamada Y, Matsuo H, Warita S, et al: Prediction of genetic risk for dyslipidemia. Genomics 90: 551-558, 2007.

6. Yamada Y, Kato K, Yoshida T, et al: Association of polymorphisms of $A B C A 1$ and ROS1 with hypertension in Japanese individuals. Int J Mol Med 21: 83-89, 2008.

7. Yamada Y, Matsuo H, Segawa T, et al: Assessment of the genetic factors for type 2 diabetes mellitus. Int J Mol Med 18: 299-308, 2006.

8. Yamada Y, Matsuo H, Watanabe S, et al: Association of a polymorphism of $C Y P 3 A 4$ with type 2 diabetes mellitus. Int $\mathrm{J}$ Mol Med 20: 703-707, 2007.
9. Lakka HM, Laaksonen DE, Lakka TA, et al: The metabolic syndrome and total and cardiovascular disease mortality in middle-aged men. JAMA 288: 2709-2716, 2002.

10. McNeill AM, Rosamond WD, Girman CJ, et al: The metabolic syndrome and 11-year risk of incident cardiovascular disease in the Atherosclerosis Risk in Communities Study. Diabetes Care 28: 385-390, 2005.

11. Boden-Albala B, Sacco RL, Lee HS, et al: Metabolic syndrome and ischemic stroke risk. Northern Manhattan Study. Stroke 39: 30-35, 2008.

12. Chen HJ, Bai CH, Yeh WT, Chiu HC and Pan WH: Influence of metabolic syndrome and general obesity on the risk of ischemic stroke. Stroke 37: 1060-1064, 2006.

13. Iso H, Sato S, Kitamura A, et al: Metabolic syndrome and the risk of ischemic heart disease and stroke among Japanese men and women. Stroke 38: 1744-1751, 2007.

14. Kurl S, Laukkanen JA, Niskanen L, et al: Metabolic syndrome and the risk of stroke in middle-aged men. Stroke 37: 806-811, 2006.

15. Najarian RM, Sullivan LM, Kannel WB, Wilson PW, D'Agostino RB and Wolf PA: Metabolic syndrome compared with type 2 diabetes mellitus as a risk factor for stroke: the Framingham Offspring Study. Arch Intern Med 166: 106-111, 2006.

16. Warlow C, Sudlow C, Dennis M, Wardlaw J and Sandercock P: Stroke. Lancet 362: 1211-1224, 2003.

17. Yamada Y, Kato K, Oguri M, et al: Association of genetic variants with atherothrombotic cerebral infarction in Japanese individuals with metabolic syndrome. Int J Mol Med 21: 801-808, 2008.

18. Sattar N, Gaw A, Scherbakova O, et al: Metabolic syndrome with and without $\mathrm{C}$-protein as a predictor of coronary heart disease and diabetes in the West of Scotland Coronary Prevention Study. Circulation 108: 414-419, 2003.

19. Ridker PM, Buring JE, Cook NR and Rifai N: C-reactive protein, the metabolic syndrome, and risk of incident cardiovascular events: an 8-year follow-up of 14719 initially healthy American women. Circulation 107: 391-397, 2003.

20. Kanazawa M, Yoshiike N, Osaka T, Numba Y, Zimmet P and Inoue $\mathrm{S}$ : Criteria and classification of obesity in Japan and AsiaOceania. Asia Pac J Clin Nutr 11: S732-S737, 2002.

21. A committee established by the Director of the National Institute of Neurological Disorders and Stroke, National Institutes of Health: Special report from the National Institute of Neurological Disorders and Stroke. Classification of Cerebrovascular Diseases III. Stroke 21: 637-676, 1990.

22. Yamada Y, Fuku N, Tanaka M, et al: Identification of CELSR1 as a susceptibility gene for ischemic stroke in Japanese individuals by a genome-wide association study. Atherosclerosis (published on line).

23. Itoh Y, Mizuki N, Shimada T, et al: High throughput DNA typing of HLA-A, -B, -C and -DRB1 loci by a PCR-SSOPLuminex method in the Japanese population. Immunogenetics 57: 717-729, 2005.

24. Knowles WJ, Bologna ML, Chasis JA, Marchesi SL and Marchesi VT: Common structural polymorphisms in human erythrocyte spectrin. J Clin Invest 73: 973-979, 1984.

25. Stabach PR and Morrow JS: Identification and characterization of beta V spectrin, a mammalian ortholog of Drosophila beta $\mathrm{H}$ spectrin. J Biol Chem 275: 21385-21395, 2000.

26. International Multiple Sclerosis Genetics Consortium (IMSGC): The expanding genetic overlap between multiple sclerosis and type I diabetes. Genes Immun 10: 11-14, 2009.

27. Âwata T, Kawasaki E, Tanaka S, et al: Association of type 1 diabetes with two Loci on $12 \mathrm{q} 13$ and $16 \mathrm{p} 13$ and the influence coexisting thyroid autoimmunity in Japanese. J Clin Endocrinol Metab 94: 231-235, 2009.

28. Todd JA, Walker NM, Cooper JD, et al: Robust associations of four new chromosome regions from genome-wide analyses of type 1 diabetes. Nat Genet 39: 857-864, 2007.

29. Hakonarson H, Grant SF, Bradfield JP, et al: A genome-wide association study identifies KIAA0350 as a type 1 diabetes gene. Nature 448: 591-594, 2007. 